\title{
Axially Symmetric Bulk Viscous String Cosmological Models in GR and Brans-Dicke Theory of Gravitation
}

\author{
V. U. M. Rao and D. Neelima \\ Department of Applied Mathematics, Andhra University, Visakhapatnam 530003, India \\ Correspondence should be addressed to V. U. M. Rao; umrao57@hotmail.com
}

Received 5 August 2013; Accepted 25 September 2013

Academic Editors: G. Bothun, H. Dehnen, and H. Zhao

Copyright ( 2013 V. U. M. Rao and D. Neelima. This is an open access article distributed under the Creative Commons Attribution License, which permits unrestricted use, distribution, and reproduction in any medium, provided the original work is properly cited.

\begin{abstract}
Axially symmetric string cosmological models with bulk viscosity in Brans-Dicke (1961) and general relativity (GR) have been studied. The field equations have been solved by using the anisotropy feature of the universe in the axially symmetric space-time. Some important features of the models, thus obtained, have been discussed. We noticed that the presence of scalar field does not affect the geometry of the space-time but changes the matter distribution, and as a special case, it is always possible to obtain axially symmetric string cosmological model with bulk viscosity in general relativity.
\end{abstract}

\section{Introduction}

Brans and Dicke [1] theory of gravitation is a natural extension of general relativity which introduces an additional scalar field $\phi$ besides the metric tensor $g_{i j}$ and dimensionless coupling constant $\omega$. The Brans-Dicke field equations for combined scalar and tensor fields (using geometrized units with $c=1, G=1$ ) are given by

$$
\begin{gathered}
G_{i j}=-8 \pi \phi^{-1} T_{i j}-\omega \phi^{-2}\left(\phi_{, i} \phi_{, j}-\frac{1}{2} g_{i j} \phi_{, k} \phi^{, k}\right) \\
-\phi^{-1}\left(\phi_{i ; j}-g_{i j} \phi_{; k}^{, k}\right), \\
\phi_{; k}^{, k}=8 \pi(3+2 \omega)^{-1} T,
\end{gathered}
$$

where $G_{i j}=R_{i j}-(1 / 2) R g_{i j}$ is an Einstein tensor, $R$ is the scalar curvature, $\omega$ is a dimension less constant, and $T_{i j}$ is the stress energy tensor of the matter. Also comma and semicolon denote partial and covariant differentiation, respectively.

Also, we have energy-conservation equation:

$$
T_{; j}^{i j}=0 .
$$

Several aspects of Brans-Dicke cosmology have been extensively investigated by many authors. Reddy and Rao $[2,3]$ have obtained field of a charged particle and static plane symmetric solution in Brans-Dicke theory. The work of Singh and Rai [4] gives a detailed survey of Brans-Dicke cosmological models discussed by several authors. Rao et al. [5] have obtained exact Bianchi type-V perfect fluid cosmological models in Brans-Dicke theory of gravitation. Tiwari [6] has obtained Bianchi type-V cosmological models with constant deceleration parameter in a scalar-tensor theory. Rao and Vinutha [7] have obtained axially symmetric cosmological models in a scalar tensor theory based on Lyra [8] Manifold. Rao et al. [9] have discussed LRS Bianchi type-I dark energy cosmological model in Brans-Dicke theory of gravitation. Rao and Neelima [10] have discussed axially symmetric space-time with strange quark matter attached to the string cloud in self-creation theory and general relativity and established that the additional condition, special law of variation of Hubble parameter proposed by Berman [11], taken by Katore and Shaikh [12] in general relativity is superfluous. Rao and Sireesha $[13,14]$ have discussed axially symmetric, Bianchi type-II, -VIII, and -IX cosmological models with strange quark matter attached to string cloud in Brans-Dicke theory of gravitation, respectively.

Cosmic strings are important in the early stages of evolution of the universe before the particle creation. Cosmic strings are one-dimensional topological defects associated 
with spontaneous symmetry breaking whose plausible production site is cosmological phase transitions in the early universe. Letelier [15], Krori et al. [16], Mahanta and Mukherjee [17], and Bhattacharjee and Baruah [18] have studied several aspects of string cosmological models in general relativity. Rao et al. [19-21] have obtained axially symmetric string, perfect fluid cosmological models, and Bianchi type-II, -VIII, and -IX magnetized cosmological models in Brans-Dicke theory of gravitation.

In order to study the evolution of the universe, many authors constructed cosmological models containing a viscous fluid. The presence of viscosity in the fluid introduces many interesting features in the dynamics of homogeneous cosmological models. The possibility of bulk viscosity leading to inflationary like solutions in general relativistic FRW models has been discussed by several authors. Rao et al. [22] have discussed anisotropic universe with cosmic strings and bulk viscosity in a scalar-tensor theory of gravitation. Rao et al. [23] have obtained Bianchi type-II, -VIII, and -IX string cosmological models with bulk viscosity in a theory of gravitation. Rao and Sireesha $[24,25]$ have studied a higherdimensional string, and Bianchi type-II, -VIII, and -IX string cosmological models with bulk viscosity, respectively, in Brans-Dicke theory of gravitation. Rao and Sireesha [26] have discussed axially symmetric string cosmological model with bulk viscosity in self-creation theory of gravitation.

In this paper, we will study axially symmetric string cosmological models with bulk viscosity in Brans-Dicke and general relativity.

\section{Axially Symmetric Metric and Field Equations}

We consider the axially symmetric space-time as

$$
d s^{2}=d t^{2}-A^{2}(t)\left[d \chi^{2}+f^{2}(\chi) d \varphi^{2}\right]-B^{2}(t) d z^{2},
$$

where $A$ and $B$ are functions of time $t$ alone while $f$ is a function of coordinate $\chi$ alone.

The energy momentum tensor for a bulk viscous fluid containing one dimensional string is

$$
\begin{gathered}
T_{i j}=(\rho+\bar{p}) u_{i} u_{j}-\bar{p} g_{i j}-\lambda x_{i} x_{j}, \\
\bar{p}=p-3 \xi H,
\end{gathered}
$$

where $p=\epsilon_{0} \rho\left(0 \leq \epsilon_{0} \leq 1\right)$. Here $\bar{p}$ is the total pressure which includes the proper pressure $p, \rho$ is the rest energy density of the system, $\lambda$ is the tension in the string, $\xi(t)$ is the coefficient of bulk viscosity, $3 \xi H$ is usually known as bulk viscous pressure, $H$ is the Hubble parameter, $u_{i}$ is the four velocity vector, and $x_{i}$ is a space-like vector which represents the anisotropic directions of the string.

Also $u^{i}$ and $x^{i}$ satisfy the equations

$$
\begin{gathered}
g_{i j} u^{i} u^{j}=1, \\
g_{i j} x^{i} x^{j}=-1, \\
u^{i} x_{i}=0 .
\end{gathered}
$$

Here we assume that the string is lying along the $z$-axis. The one-dimensional strings are assumed to be loaded with particles and the particle energy density is

$$
\rho_{p}=\rho-\lambda \text {. }
$$

The components of energy momentum tensor are

$$
T_{1}^{1}=T_{2}^{2}=-\bar{p}, \quad T_{3}^{3}=\lambda-\bar{p}, \quad T_{4}^{4}=\rho,
$$

where $\rho, \lambda$, and $\bar{p}$ are functions of time $t$ only.

\section{Cosmological Models}

Now with the help of (5) to (10), the field equations (1) for the metric (4) can be written as

$$
\begin{gathered}
\frac{\ddot{A}}{A}+\frac{\ddot{B}}{B}+\frac{\dot{A} \dot{B}}{A B}+\frac{\omega \dot{\phi}^{2}}{2 \phi^{2}}+\frac{\ddot{\phi}}{\phi}+\frac{\dot{\phi}}{\phi}\left(\frac{\dot{A}}{A}+\frac{\dot{B}}{B}\right)=-\frac{8 \pi}{\phi} \bar{p}, \\
2 \frac{\ddot{A}}{A}+\frac{\dot{A}^{2}}{A^{2}}-\frac{f^{\prime \prime}}{A^{2} f}+\frac{\omega \dot{\phi}^{2}}{2 \phi^{2}}+\frac{\ddot{\phi}}{\phi}+2 \frac{\dot{\phi}}{\phi} \frac{\dot{A}}{A}=\frac{8 \pi}{\phi}(\lambda-\bar{p}), \\
\frac{\dot{A}^{2}}{A^{2}}+2 \frac{\dot{A} \dot{B}}{A B}-\frac{f^{\prime \prime}}{A^{2} f}-\frac{\omega \dot{\phi}^{2}}{2 \phi^{2}}+\frac{\dot{\phi}}{\phi}\left(2 \frac{\dot{A}}{A}+\frac{\dot{B}}{B}\right)=\frac{8 \pi}{\phi} \rho, \\
\ddot{\phi}+\dot{\phi}\left(2 \frac{\dot{A}}{A}+\frac{\dot{B}}{B}\right)=\frac{8 \pi}{(3+2 \omega)}(\rho+\lambda-3 \bar{p}), \\
\dot{\rho}+(\rho+\bar{p})\left(2 \frac{\dot{A}}{A}+\frac{\dot{B}}{B}\right)-\lambda \frac{\dot{B}}{B}=0 .
\end{gathered}
$$

Here the overhead dot and dash denote ordinary differentiation with respect to $t$ and $\chi$, respectively.

From (11) to (13), we can observe that it is possible to separate the terms of $f(\chi)$ to one side and the terms of $A(t), B(t), \phi(t), \rho(t), \bar{p}(t)$, and $\lambda(t)$ to another side. Hence, we can take that each part is equal to a constant. So,

$$
\frac{f^{\prime \prime}}{f}=k^{2}, \quad k^{2} \text { is a constant. }
$$

If $k=0$, then $f(\chi)=c_{1} \chi+c_{2}, \chi>0$, where $c_{1}$ and $c_{2}$ are integrating constants.

Without loss of generality, by taking $c_{1}=1$ and $c_{2}=0$, we have $f(\chi)=\chi$.

Now the field equations (11) to (15) will reduce to

$$
\begin{gathered}
\frac{\ddot{A}}{A}+\frac{\ddot{B}}{B}+\frac{\dot{A} \dot{B}}{A B}+\frac{\omega \dot{\phi}^{2}}{2 \phi^{2}}+\frac{\ddot{\phi}}{\phi}+\frac{\dot{\phi}}{\phi}\left(\frac{\dot{A}}{A}+\frac{\dot{B}}{B}\right)=-\frac{8 \pi}{\phi} \bar{p}, \\
2 \frac{\ddot{A}}{A}+\frac{\dot{A}^{2}}{A^{2}}+\frac{\omega \dot{\phi}^{2}}{2 \phi^{2}}+\frac{\ddot{\phi}}{\phi}+2 \frac{\dot{\phi}}{\phi} \frac{\dot{A}}{A}=\frac{8 \pi}{\phi}(\lambda-\bar{p}), \\
\frac{\dot{A}^{2}}{A^{2}}+2 \frac{\dot{A} \dot{B}}{A B}-\frac{\omega \dot{\phi}^{2}}{2 \phi^{2}}+\frac{\dot{\phi}}{\phi}\left(2 \frac{\dot{A}}{A}+\frac{\dot{B}}{B}\right)=\frac{8 \pi}{\phi} \rho, \\
\ddot{\phi}+\dot{\phi}\left(2 \frac{\dot{A}}{A}+\frac{\dot{B}}{B}\right)=\frac{8 \pi}{(3+2 \omega)}(\rho+\lambda-3 \bar{p}), \\
\dot{\rho}+(\rho+\bar{p})\left(2 \frac{\dot{A}}{A}+\frac{\dot{B}}{B}\right)-\lambda \frac{\dot{B}}{B}=0 .
\end{gathered}
$$


The field equations (17) to (20) are four independent equations with six unknowns $A, B, \phi, \rho, \bar{p}$, and $\lambda$.

From (17) to (20), we get

$$
\omega\left[\frac{\ddot{\phi}}{\phi}-\frac{1}{2} \frac{\dot{\phi}^{2}}{\phi^{2}}+\frac{\dot{\phi}}{\phi}\left(2 \frac{\dot{A}}{A}+\frac{\dot{B}}{B}\right)\right]=2 \frac{\ddot{A}}{A}+2 \frac{\dot{A} \dot{B}}{A B}+\frac{\ddot{B}}{B}+\frac{\dot{A}^{2}}{A^{2}} .
$$

In order to get a deterministic solution, we take the following plausible physical condition: the shear scalar $\sigma$ is proportional to scalar expansion $\theta$, which leads to the linear relationship between the metric potentials $A$ and $B$, that is,

$$
A=B^{n}, \quad n \neq 0,
$$

where $n$ is an arbitrary constant.

From (22) and (23), we get

$$
\omega\left[\frac{\ddot{\phi}}{\phi}-\frac{1}{2} \frac{\dot{\phi}^{2}}{\phi^{2}}+(2 n+1) \frac{\dot{\phi}}{\phi} \frac{\dot{B}}{B}\right]=(2 n+1) \frac{\ddot{B}}{B}+3 n^{2} \frac{\dot{B}^{2}}{B^{2}} \text {. }
$$

From (24) and (23), we get

$$
\begin{gathered}
B=\left[k_{3}\left(k_{1} t+k_{2}\right)\right]^{1 / k_{3}}, \\
A=\left[k_{3}\left(k_{1} t+k_{2}\right)\right]^{n / k_{3}}, \quad \text { where } k_{3}=\frac{n^{2}+n+1}{n+1}, n \neq-1,
\end{gathered}
$$

$k_{1} \neq 0$ and $k_{2}$ are arbitrary constants, and

$$
\phi=\left(k_{1} t+k_{2}\right)^{r}
$$

where $r=n(n+2) /\left(n^{2}+n+1\right)[-1 \pm \sqrt{1+2(n+1) / \omega(n+2)}]$.

Since Brans-Dicke theory has to go to Einstein theory in all respects when $\omega \rightarrow \infty, \phi=$ constant, then $r$ should be of the form

$$
r=\frac{n(n+2)}{\left(n^{2}+n+1\right)}\left[-1+\sqrt{1+\frac{2(n+1)}{\omega(n+2)}}\right] .
$$

The metric (4) can now be written as

$$
\begin{aligned}
d s^{2}= & d t^{2}-\left[k_{3}\left(k_{1} t+k_{2}\right)\right]^{2 n / k_{3}}\left[d \chi^{2}+\chi^{2} d \varphi^{2}\right] \\
& -\left[k_{3}\left(k_{1} t+k_{2}\right)\right]^{2 / k_{3}} d z^{2} .
\end{aligned}
$$

From (19), we get the string energy density

$$
\rho=\frac{k_{1}^{2}}{8 \pi k_{3}^{2}}\left[n(n+2)+(2 n+1) r k_{3}-\frac{\omega}{2} r^{2} k_{3}^{2}\right]\left(k_{1} t+k_{2}\right)^{r-2} \text {. }
$$

From (17), we get the total pressure

$$
\begin{aligned}
\bar{p}=\frac{k_{1}^{2}}{8 \pi k_{3}^{2}}\left[(n+1)\left(k_{3}-1\right)-n^{2}+r(1-r) k_{3}^{2}\right. \\
\left.-(n+1) r k_{3}-\frac{\omega}{2} r^{2} k_{3}^{2}\right]\left(k_{1} t+k_{2}\right)^{r-2} .
\end{aligned}
$$

From (17) and (18), we get the string tension density

$$
\lambda=\frac{k_{1}^{2}(n-1)}{8 \pi k_{3}^{2}}\left[(2 n+1)+(r-1) k_{3}\right]\left(k_{1} t+k_{2}\right)^{r-2} .
$$

The proper pressure is given by

$$
\begin{array}{r}
p=\varepsilon_{0} \rho=\frac{\varepsilon_{0} k_{1}^{2}}{8 \pi k_{3}^{2}}\left[n(n+2)+(2 n+1) r k_{3}\right. \\
\left.-\frac{\omega}{2} r^{2} k_{3}^{2}\right]\left(k_{1} t+k_{2}\right)^{r-2} .
\end{array}
$$

The coefficient of bulk viscosity is given by

$$
\begin{aligned}
\xi= & \frac{k_{1}}{8 \pi(2 n+1) k_{3}} \\
& \times\left[\begin{array}{c}
n^{2}\left(1+\varepsilon_{0}\right)+2 n \varepsilon_{0}+\frac{\omega}{2} r^{2} k_{3}^{2}\left(1-\varepsilon_{0}\right) \\
+r k_{3}\left[(2 n+1) \varepsilon_{0}+(r-1) k_{3}\right. \\
+(n+1)]+(n+1)\left(1-k_{3}\right)
\end{array}\right] \\
& \times\left(k_{1} t+k_{2}\right)^{r-1} .
\end{aligned}
$$

Thus, the metric (28) together with (26) and (29) to (33) constitutes axially symmetric string cosmological model with bulk viscosity in Brans-Dicke scalar-tensor theory gravitation where the constants are related by

$$
\left(3 n^{2}+2 n+1\right)+k_{3}\left[\frac{\omega}{2} r k_{3}(2-r)-(2 n+1)(1+\omega r)\right]=0 .
$$

\section{Axially Symmetric String Cosmological Models with Bulk Viscosity in General Relativity}

It is interesting to note that when $\omega \rightarrow \infty, r=0$, and hence from (26), we get $\phi=$ constant.

From (19), we get the string energy density

$$
\rho=\frac{k_{1}^{2}}{8 \pi k_{3}^{2}}[n(n+2)]\left(k_{1} t+k_{2}\right)^{-2} .
$$

From (17), we get the total pressure

$$
\bar{p}=\frac{k_{1}^{2}}{8 \pi k_{3}^{2}}\left[(n+1)\left(k_{3}-1\right)-n^{2}\right]\left(k_{1} t+k_{2}\right)^{-2} .
$$

From (17) and (18), we get the string tension density

$$
\lambda=\frac{k_{1}^{2}(n-1)}{8 \pi k_{3}^{2}}\left[(2 n+1)-k_{3}\right]\left(k_{1} t+k_{2}\right)^{-2} .
$$

The proper pressure is given by

$$
p=\varepsilon_{0} \rho=\frac{\varepsilon_{0} k_{1}^{2}}{8 \pi k_{3}^{2}}[n(n+2)]\left(k_{1} t+k_{2}\right)^{-2} \text {. }
$$


The coefficient of bulk viscosity is given by

$$
\begin{aligned}
\xi=\frac{k_{1}}{8 \pi(2 n+1) k_{3}} & {\left[n^{2}\left(1+\varepsilon_{0}\right)+2 n \varepsilon_{0}\right.} \\
& \left.+(n+1)\left(1-k_{3}\right)\right]\left(k_{1} t+k_{2}\right)^{-1} .
\end{aligned}
$$

Then the present model (28) together with (35) to (39) represents axially symmetric string cosmological model with bulk viscosity in Einstein's theory of gravitation. Also the solution identically satisfies the divergence equation (21).

\section{Some Other Important Properties of the Models}

The spatial volume of the model (28) is given by

$$
\begin{array}{r}
V=\sqrt{-g}=\chi\left[k_{3}\left(k_{1} t+k_{2}\right)^{(2 n+1) / k_{3}}\right], \\
k_{3}=\frac{n^{2}+n+1}{n+1} .
\end{array}
$$

The expansion scalar $\theta$, calculated for the flow vector $u^{i}$, is given by

$$
\theta=\frac{(2 n+1)(n+1) k_{1}}{\left(n^{2}+n+1\right)\left(k_{1} t+k_{2}\right)}
$$

The shear scalar $\sigma$ is given by

$$
\sigma^{2}=\frac{1}{2} \sigma_{i j} \sigma^{i j}=\frac{7}{18} \frac{(2 n+1)^{2}(n+1)^{2} k_{1}^{2}}{\left(n^{2}+n+1\right)^{2}\left(k_{1} t+k_{2}\right)^{2}} .
$$

The mean Hubble parameter $H$ is given by

$$
H=\frac{(2 n+1)(n+1) k_{1}}{3\left(n^{2}+n+1\right)\left(k_{1} t+k_{2}\right)} .
$$

The mean anisotropy parameter $A_{m}$ is given by

$$
A_{m}=\frac{1}{3} \sum_{i=1}^{3}\left(\frac{H_{i}-H}{H}\right)^{2}=\frac{2(n-1)^{2}}{(2 n+1)^{2}} .
$$

The density parameter $\Omega$ is given by

$$
\begin{aligned}
\Omega= & \frac{\rho}{3 H^{2}} \\
= & \frac{\left[3\left[n(n+2)+(2 n+1) r k_{3}-(\omega / 2) r^{2} k_{3}^{2}\right]\right.}{16 \pi(n-1)^{2}} \\
& \left.\quad \times\left(k_{1} t+k_{2}\right)^{r}\right] A_{m} .
\end{aligned}
$$

The deceleration parameter is given by

$$
q=-3 \theta^{-2}\left[\theta_{, \alpha} u^{\alpha}+\frac{1}{3} \theta^{2}\right]=\frac{n^{2}+2}{(2 n+1)(n+1)}, \quad n \neq-1,-\frac{1}{2} .
$$

The tensor of rotation $w_{i j}=u_{i, j}-u_{j, i}$ is identically zero and hence, this universe is nonrotational.

\section{Conclusions}

In this paper, we have presented axially symmetric string cosmological models with bulk viscosity in Brans-Dicke theory of gravitation and general relativity. We observe that model (28) has no singularity at $t=-k_{2} / k_{1}$ for $n>0$ and at this point the proper volume will be zero, whereas when $t \rightarrow \infty$, the spatial volume becomes infinitely large. At $t=-k_{2} / k_{1}$, the expansion scalar $\theta$ and shear scalar $\sigma$ tend to infinity whereas when $t \rightarrow \infty$, expansion scalar $\theta$ and shear scalar $\sigma$ tend to zero. The Hubble parameter $H$ increases with time for $n>0$. Equation (45) shows that the density parameter $\Omega$ depends on the anisotropy parameter $A_{m}$. Also, since the mean anisotropy parameter $A_{m} \neq 0$, the model does not approach isotropy for $n \neq 1$. But for $n=1$, the mean anisotropy parameter is zero and hence, the model (28) will become isotropic. Also for $n=1$ the models presented here represent only bulk viscous isotropic cosmological models. It is interesting to note that the models represent accelerating universes for $-1<n<-1 / 2$ and conformed to the well known fact that the scalar field and the bulk viscosity will play a vital role in getting an accelerated universe. Also recent observations of type Ia supernovae (SN Ia), Perlmutter et al. [27] and Riess et al. [28-30] proved that the decelerating parameter of the universe is in the range $-1 \leq q \leq 0$, and the present day universe is undergoing accelerated expansion. Finally, we noticed that the presence of scalar field in BransDicke theory does not affect the geometry of the spacetime but changes the matter distribution in comparison with Einstein theory, and as a special case, it is always possible to obtain axially symmetric string cosmological model with bulk viscosity in general relativity.

\section{Acknowledgment}

The second author (D. Neelima) is grateful to the Department of Science and Technology (DST), New Delhi, India, for providing INSPIRE fellowship.

\section{References}

[1] C. Brans and R. H. Dicke, "Mach's principle and a relativistic theory of gravitation," Physical Review, vol. 124, no. 3, pp. 925935, 1961.

[2] D. R. K. Reddy and V. U. M. Rao, "Field of a charged particle in Brans-Dicke theory of gravitation," Journal of Physics A, vol. 14, p. 1973, 1981.

[3] D. R. K. Reddy and V. U. M. Rao, "Field of a charged particle in the presence of scalar meson fields in general relativity," The Journal of the Australian Mathematical Society B, vol. 24, p. 461, 1983.

[4] T. Singh and L. N. Rai, "Scalar-tensor theories of gravitation: foundations and prospects," General Relativity and Gravitation, vol. 15, no. 9, pp. 875-902, 1983.

[5] V. U. M. Rao, T. Vinutha, M. V. Shanthi, and K. V. S. Sireesha, "Exact Bianchi type-V perfect fluid cosmological models in Brans-Dicke theory of gravitation," Astrophysics and Space Science, vol. 315, no. 1-4, pp. 211-214, 2008. 
[6] R. K. Tiwari, "Bianchi type-V cosmological models with constant deceleration parameter in scalar-tensor theory," Fizika $B$, vol. 18, no. 1, pp. 9-18, 2009.

[7] V. U. M. Rao and T. Vinutha, "Axially symmetric cosmological models in a scalar tensor theory based on Lyra manifold," Astrophysics and Space Science, vol. 319, no. 2-4, pp. 161-167, 2009.

[8] G. Lyra, "Über eine modifikation der riemannschen geometrie," Mathemastische Zeitschrift, vol. 54, no. 1, pp. 52-64, 1951.

[9] V. U. M. Rao, M. Vijaya Santhi, T. Vinutha, and G. Sreedevi Kumari, "LRS Bianchi Type-I dark energy cosmological model in Brans-Dicke theory of gravitation," International Journal of Theoretical Physics, vol. 51, no. 10, pp. 3303-3310, 2012.

[10] V. U. M. Rao and D. Neelima, "Axially symmetric space-time with strange Quark matter attached to string cloud in self creation theory and general relativity," International Journal of Theoretical Physics, vol. 52, no. 2, pp. 354-361, 2013.

[11] M. S. Berman, "A special law of variation for Hubble's parameter," Il Nuovo Cimento B Series 11, vol. 74, no. 2, pp. 182-186, 1983.

[12] S. D. Katore and A. Y. Shaikh, "Cosmological model with strange Quark matter attached to cosmic string for axially symmetric space-time," International Journal of Theoretical Physics, vol. 51, no. 6, pp. 1881-1888, 2012.

[13] V. U. M. Rao and K. V. S. Sireesha, "Axially symmetric spacetime with strange Quark matter attached to string cloud in Brans-Dicke theory of gravitation," International Journal of Theoretical Physics, vol. 52, no. 4, pp. 1052-1060, 2013.

[14] V. U. M. Rao and K. V. S. Sireesha, "Bianchi Type-II, VIII \& IX cosmological models with strange Quark matter attached to string cloud in Brans-Dicke and general theory of gravitation," International Journal of Theoretical Physics, vol. 52, no. 4, pp. 1240-1249, 2013.

[15] P. S. Letelier, “String cosmologies"” Physical Review D, vol. 28, no. 10, pp. 2414-2419, 1983.

[16] K. D. Krori, T. Chaudhuri, C. R. Mahanta, and A. Majumdar, "Some exact solutions in string cosmology," General Relativity and Gravitation, vol. 22, no. 2, pp. 123-130, 1990.

[17] P. Mahanta and A. Mukherjee, "String models in Lyra geometry," Indian Journal of Pure and Applied Mathematics, vol. 32, no. 2, pp. 199-204, 2001.

[18] R. Bhattacharjee and K. K. Baruah, "String cosmologies with a scalar field," Indian Journal of Pure and Applied Mathematics, vol. 32, no. 1, pp. 47-53, 2001.

[19] V. U. M. Rao, T. Vinutha, and K. V. S. Sireesha, "Axially symmetric string cosmological models in Brans-Dicke theory of gravitation," Astrophysics and Space Science, vol. 323, no. 4, pp. 401-405, 2009.

[20] V. U. M. Rao and K. V. S. Sireesha, "Axially symmetric perfect fluid cosmological models in Brans-Dicke scalar tensor theory of gravitation," The African Review of Physics, vol. 7, Article ID 0042, 2012.

[21] V. U. M. Rao and M. Vijaya Santhi, "Bianchi type-II, VIII \& IX perfect fluid magnetized cosmological models in Brans-Dicke theory of gravitation," Astrophysics and Space Science, vol. 337, no. 1, pp. 387-392, 2012.

[22] V. U. M. Rao, G. Sree Devi Kumari, and K. V. S. Sireesha, "Anisotropic universe with csomic strings and bulk viscosity in a scalar-tensor theory of gravitation," Astrophysics and Space Science, vol. 335, pp. 635-638, 2011.
[23] V. U. M. Rao, K. V. S. Sireesha, and M. Vijaya Santhi, "Bianchi Types II, VIII, and IX string cosmological models with bulk viscosity in a theory of gravitation," ISRN Mathematical Physics, vol. 2012, Article ID 341612, 15 pages, 2012.

[24] V. U. M. Rao and K. V. S. Sireesha, "A higher-dimensional string cosmological model in a scalar-tensor theory of gravitation," The European Physical Journal Plus, vol. 127, article 33, 2012.

[25] V. U. M. Rao and K. V. S. Sireesha, "Bianchi Types II, VIII and IX string cosmological models with bulk viscosity in bransdicke theory of gravitation," International Journal of Theoretical Physics, vol. 51, no. 10, pp. 3013-3020, 2012.

[26] V. U. M. Rao and K. V. S. Sireesha, "Axially symmetric string cosmological model with bulk viscosity in self-creation theory of gravitation," The European Physical Journal Plus, vol. 127, article 49, 2012.

[27] S. Perlmutter, G. Aldering, G. Goldhaber et al., "Measurements of $\mathrm{O}$ and $\Lambda$ from 42 high-redshift supernovae," The Astrophysical Journal, vol. 517, no. 2, p. 565, 1999.

[28] A. G. Reiss, A. V. Filippenko, P. Challis et al., "Observational evidence from supernovae for an accelerating universe and a cosmological constant," The Astrophysical Journal, vol. 116, pp. 1009-1038, 1998.

[29] A. G. Riess, R. P. Kirshner, B. P. Schmidt et al., "BVRI light curves for 22 type Ia supernovae," The Astronomical Journal, vol. 117, p. 707, 1999.

[30] A. G. Riess, L.-G. Strolger, J. Tonry et al., “Type Ia supernova discoveries at $z>1$ from the Hubble Space Telescope: evidence for past deceleration and constraints on dark energy evolution," The Astrophysical Journal, vol. 607, p. 665, 2004. 

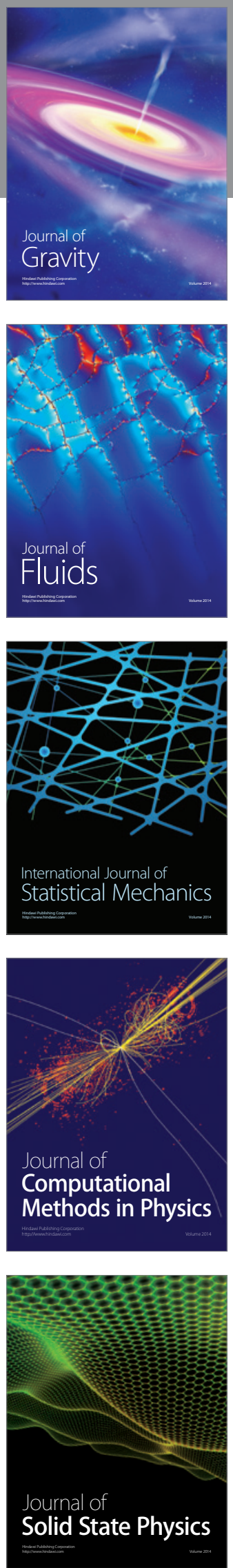

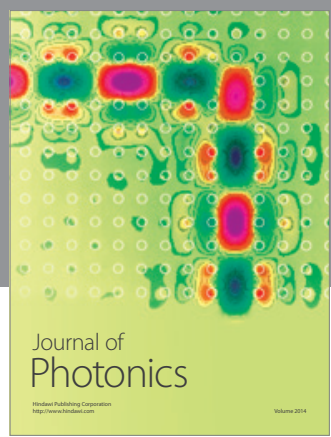

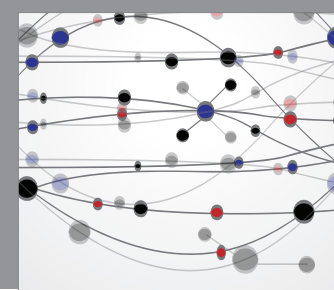

The Scientific World Journal

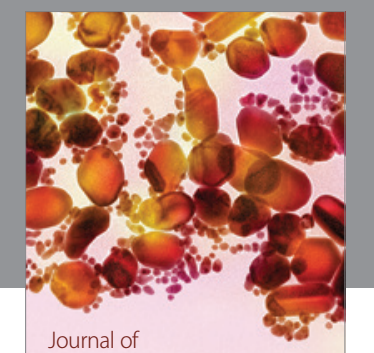

Soft Matter
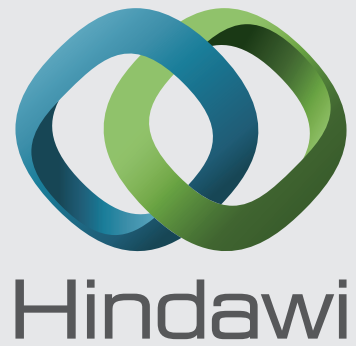

Submit your manuscripts at

http://www.hindawi.com
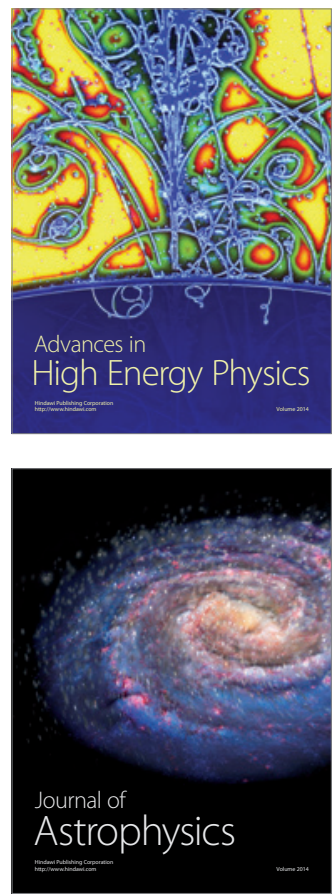
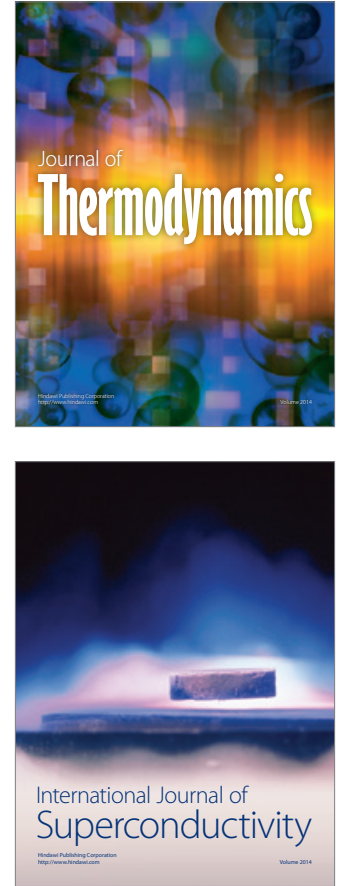
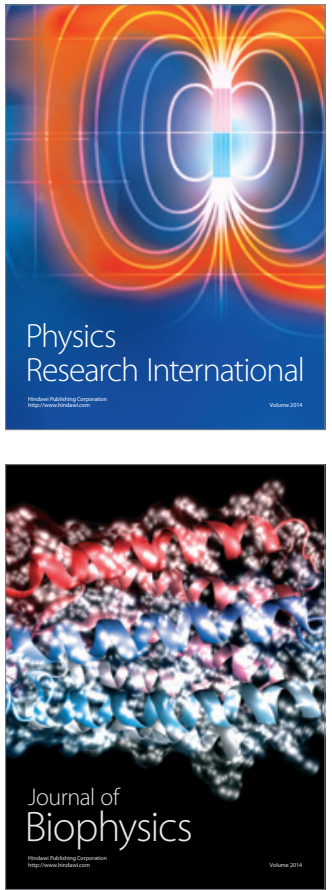
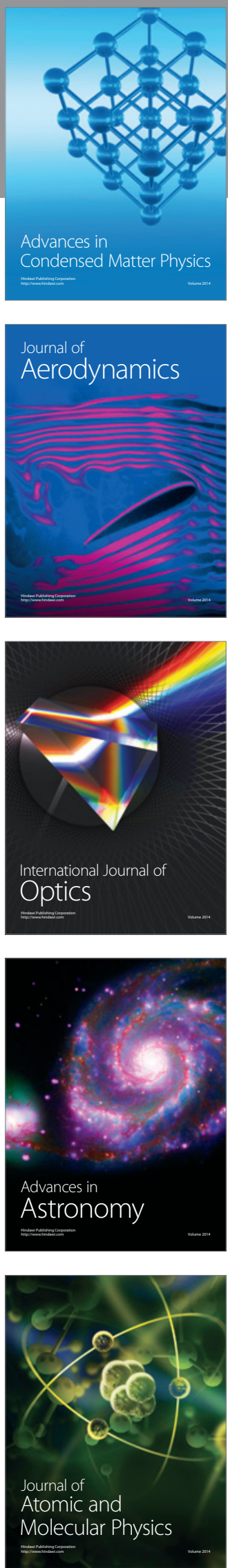